\title{
Nuevo marco regulatorio y competencia: retos del sistema crediticio europeo en la era fintech
}

\author{
Miguel Ángel Bernal Alonso \\ Javier Santacruz Cano \\ Instituto de Estudios Bursátiles
}

\section{Resumen}

Los bancos y aseguradoras que operan en Europa se enfrentan a una serie de desafios en su actividad en un escenario actual de bajo crecimiento del PIB, envejecimiento de la población, bajos tipos de interés y el protagonismo de Asia en el equilibrio mundial. En este sentido, la aparición de la industria fintech supone más competencia en este sector. En el presente estudio, partimos de la regulación básica (Basilea III y MiFID II) junto con su repercusión en la rentabilidad de los capitales, para examinar las claves del nuevo modelo de negocio que trae la industria Fintech y los desafios que plantea en materia regulatoria, de recursos propios y riesgos.

Palabras clave: bancos, seguros, eficiencia, regulación, fintech, $R O E$, recursos propios.

Clasificación JEL: G21, G22, G28.

\section{Abstract}

European banks and insurance companies are facing several challenges in their proper business under a complicated scenario with low GDP growth, ageing, low interest rates and a growing role of Asia in the economy. Furthermore, the appearance of the fintech industry means a new competitor in this sector. In our study, we start with the basic regulation principles (Basel III and MiFID II) and their impact on capital profitability, in order to examine the key points of the new banking and insurance model that comes with the fintech industry and challenges over the business model, regulation, capital and risk management.

Keywords: banks, insurance companies, cost-to-income ratio, regulation, fintech, ROE, equity.

JEL classification: G21, G22, G28.

\section{Introducción}

Ante el nuevo marco regulatorio que se está confeccionando para bancos y compañías aseguradoras, se abren una serie de nuevos retos a abordar por parte de ambos sectores. Estos retos crean una mayor incertidumbre ante la atonía de crecimiento en Europa, el envejecimiento de la población, bajos tipos de interés y desplazamiento hacia Asia-Pacífico de la atención económica. Queda, por tanto, en entredicho la generación de rentabilidad para el accionista (ROE) e incluso el modelo de negocio vigente. 
Como añadido al modelo de negocio imperante hasta el momento, las denominadas «empresas fintech» o tecnología financiera, irrumpen como nuevos actores y competencia directa de este tipo de entidades. En este estudio, evaluaremos el impacto de estas nuevas variables teniendo presente que no existe precedente histórico de este conjunto de cambios en la banca, por lo que es muy difícil hacer comparativas anteriores y en un estadio inicial de la literatura sobre tecnología financiera.

Los estudios más recientes están elaborados por firmas de consultoría, las cuales evalúan diferentes escenarios de desarrollo de las fintech. Sin duda, uno de los elementos de interés prioritario en la extensión de las fintech es la regulación que a medio plazo se establecerá sobre estos nuevos canales y hasta qué punto este hecho puede frenar su crecimiento. De esta cuestión se encargan unos pocos autores que examinan la implementación de nuevas normas regulatorias (Treleaven, 2015) o que hacen una simulación de cómo estaría la industria fintech reglada si los reguladores siguieran los mismos principios que están aplicando a los bancos tradicionales (Kaminska, 2015).

La mayor parte de los análisis provienen de estudios profesionales con enfoque top-down (Accenture, 2015) en el que subrayan la difícil convivencia entre un modelo bancario que entienden obsoleto y uno disruptivo y masivo como las fintech. Pero también encontramos a otros autores que intentan conciliar los dos modelos de hacer banca para poder salvar la brecha generacional (McMannus, 2016). Precisamente, los estudios de Wharton Fintech hacen hincapié en la adaptación de las estructuras bancarias a una demanda que en las economías desarrolladas tiende a ser más longeva, más selectiva y con una cultura financiera más amplia. Aquí es donde se suele relacionar fintech con la denominada «generación Millenial».

A partir de estos elementos, comenzamos con una breve descripción de la situación de la banca europea en medio de un nuevo marco regulatorio de Unión Bancaria. Posteriormente, se analiza la repercusión que Basilea III y la reglamentación de mercados e instrumentos financieros (MiFID II) tendrá para la rentabilidad (ROE), nivel de ingresos y gastos, eficiencia, así como volumen de capital a ampliar. En este aspecto, la solución basada en la historia sería a acometer un proceso de reducción de costes. Por último, entramos a analizar la competencia de la industria fintech y los desafíos que estos últimos tienen en materia: regulatoria, recursos propios, confianza de los clientes y riesgo sistémico que conllevarán.

Finalmente, ante esta nueva situación, veremos desarrollos y claves en cuanto a lo que entendemos como mercados y actividad financiera, contribución al crecimiento europeo e impacto sobre la estabilidad financiera a nivel mundial. En especial, merece particular atención la influencia que tienen los cambios en el modelo bancario sobre los mercados financieros, cada vez más estrechamente correlacionados con las decisiones de política monetaria y cómo estas se trasladan a los diferentes mercados (divisas, materias primas, renta fija y renta variable). 


\section{Situación actual de la economía y banca en la Unión Europea}

Los bancos europeos llevan desde hace tiempo siendo una fuente de preocupación económica y financiera a nivel global. En Europa, además, la actual situación de las entidades pueden ser un lastre para el crecimiento económico de la Eurozona, dada la preocupación que existe tanto sobre la solvencia de algunas entidades, así como la baja rentabilidad que parece desprenderse de la proyección de previsiones de sus cuentas de resultados para los próximos ejercicios.

La preocupación por el sector se inició con el estallido de la burbuja de crédito a partir del ejercicio 2008. Desde aquel momento, el punto de mira se puso en la solvencia de las entidades, así como la de algunos sistemas crediticios que comenzaron a evidenciar problemas estructurales: Irlanda, Portugal, Grecia, España o Chipre. De todos es conocido que todos estos países necesitaron de actuaciones por parte de la Unión Europea para sanear y restablecer la solvencia.

Ha pasado ya tiempo desde las últimas actuaciones, concretamente en el verano de 2013, con varios bancos chipriotas, donde por primera vez se llevó a cabo una reestructuración y saneamiento bajo el esquema bail-in o reestructuración privada, frente a los anteriores esquemas bail-out o de reestructuración pública. Estos fueron, como se verá más adelante, los primeros pasos para la Unión Bancaria y la nueva forma de abordar los problemas de solvencia que tuvieran las entidades financieras a medio y largo plazo en el marco de una supervisión unificada.

Sin embargo, todo parece indicar que aún estamos lejos de haber solucionado la situación. Ahora mismo dos son los frentes abiertos: por un lado, la solvencia de algunos bancos, especialmente los italianos; por otro lado, la rentabilidad de los modelos de negocio implementados en el sector, donde la sobrecapacidad de algunas entidades así como la irrupción de la tecnología, comprometen seriamente la rentabilidad del capital. Esta baja rentabilidad, además, se ve afectada por un entorno económico en Europa de bajo crecimiento e inflación inexistente, donde además la respuesta positiva a la salida de Reino Unido de la Unión Europea, brexit, hace más compleja aún más si cabe la situación por la incertidumbre que ha traído.

Un ejemplo muy ilustrativo lo tenemos en la evolución bursátil del sector. Para ello, tomamos como referencia el Índice Stoxx Europe 600 Banks EUR (Ticker: SX7E), índice que recoge la evolución de los 30 bancos principales cotizados en Europa y pertenecientes a diferentes países: 


\section{GRÁFICO 1}

\section{EVOLUCIÓN DEL ÍNDICE STOXX EUROPE 600 BANKS (En euros)}

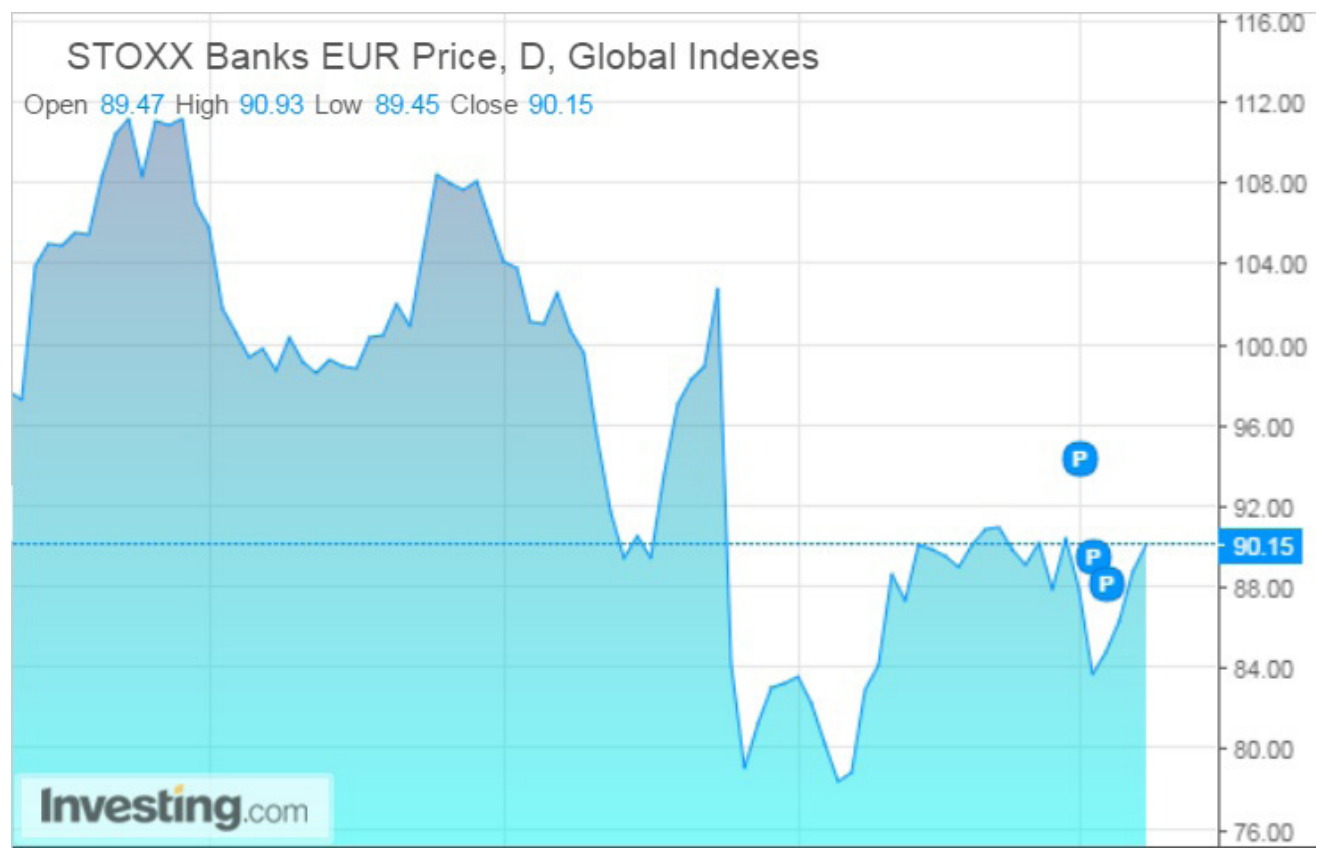

FUENTE: Investing.com.

Como puede observarse, la cotización de los treinta principales bancos europeos se encuentra a niveles similares a los del ejercicio 2012, momento álgido de los problemas bancarios, pues en junio de ese año el Gobierno español pidió ayuda financiera a la Unión Europea y estos pusieron a disposición de su economía una línea de crédito de hasta 100.000 millones de euros, prácticamente el 10 por 100 del PIB de España, aún cuando las necesidades de financiación se cifraron en aproximadamente 40.000 millones de euros.

Más allá de los problemas de solvencia de algunos bancos o sistemas crediticios concretos (lo cual no es objetivo de este estudio) conviene centrarse en los problemas del modelo de negocio y rentabilidad. En este sentido, los problemas de negocio de la banca no se deben a una única causa, sino a la conjunción de una serie de factores tanto externos como internos. Entre ellos, los bancos deben convivir con una ralentización económica y financiera tanto a nivel europeo como mundial, donde además el Brexit genera una gran incertidumbre y preocupación con la incidencia que eso tiene en el crecimiento, tal como señalábamos anteriormente. 
El FMI así lo recoge en su último informe y precisamente achaca a la situación de Reino Unido la rebaja en julio de 2016 de sus perspectivas de crecimiento mundial (WEO, por su acrónimo inglés). En el citado informe, la institución rebajó una décima las cifras de crecimiento de PIB a nivel mundial y también de la Eurozona. Así, para 2016, sitúa el crecimiento en el 3,1 por 100 para la economía global y del 1,6 por 100 para la Eurozona, mientras que sus previsiones para 2017 son del 3,4 por 100 para el mundo y del 1,4 por 100 para la Eurozona, debido a la incertidumbre por el Brexit (la salida del Reino Unido de la Unión Europea) y la efectividad de las medidas monetarias puestas en marcha en 2015 por el Banco Central Europeo (BCE) ${ }^{1}$.

Es evidente que esta situación de debilidad económica repercute en una demanda débil de crédito y, por tanto, una presión sobre sus márgenes de intermediación. Cierto es que las medidas no ortodoxas llevadas a cabo por el BCE, han aumentado de forma notable la base monetaria en el área de su influencia; sin embargo la concesión de crédito continúa siendo aún bastante baja. Incluso en algunos países como en España, el efecto neto del saldo crediticio es incluso de caída. Por tanto, y como es conocido, las perspectivas de un entorno económico de semiestancamiento e inflación muy baja no parece que pueda incentivar la demanda de crédito lo suficiente como para aumentar los resultados positivos.

\section{GRÁFICO 2}

EVOLUCIÓN DE LA TASA INTERANUAL DE CRECIMIENTO DEL CRÉDITO A RESIDENTES (SECTOR PRIVADO) EN ESPAÑA

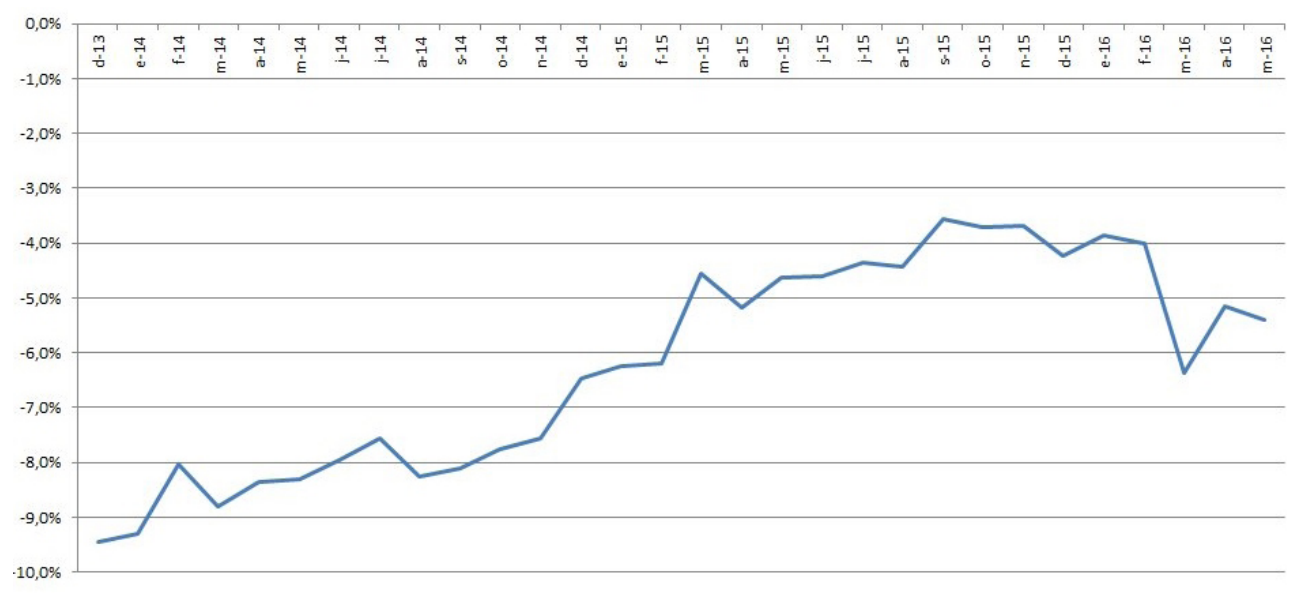

FUENTE: Elaboración propia. Banco de España.

${ }^{1}$ En la Zona Euro, la tendencia es hacia más estímulos monetarios en forma de compra de activos públicos y privados y poner más en negativo la facilidad marginal de depósito. La ampliación del programa de compra de activos hasta 80.000 millones de euros junto a la habilitación de compra de títulos de renta fija corporativa, está suponiendo un estímulo para el ritmo de emisiones de títulos de renta fija como titulizaciones y Project bonds (véase BERNAL y SANTACRUZ, 2015). 
Pero es que además la situación macroeconómica incide en la política monetaria del BCE, no solo de forma no ortodoxa, sino también en materia de tipos de interés. En el siguiente gráfico, extraído del Boletín Económico del Banco de España, correspondiente a junio de 2016, puede observarse que desde hace ya tiempo los principales tipos tanto de referencia oficial como del mercado interbancario se encuentran en terreno nulo o negativo, gracias a las medidas de expansión de la liquidez, cuyo objetivo es restablecer el mecanismo de transmisión de la política monetaria.

\section{GRÁFICO 3}

\section{OPERACIONES PRINCIPALES DE FINANCIACIÓN DEL EUROSISTEMA Y MERCADO INTERBANCARIO EN LA ZONA EURO}
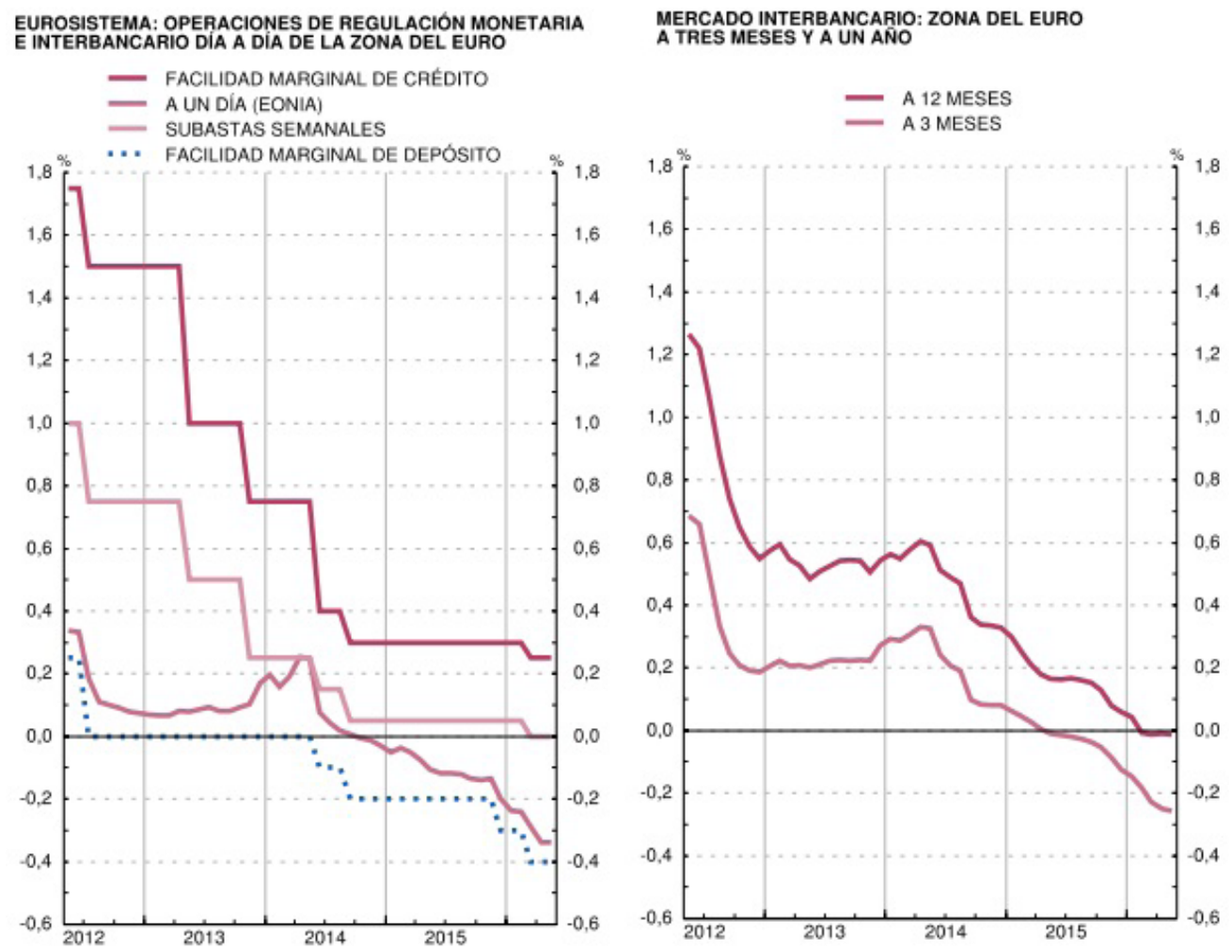

NOTA: a Hasta diciembre de 1998, se han calculado ponderando los tipos de interés nacionales por el PIB.

FUENTE: BCE (cuadernos 1 a 8 ).

Esta combinación de baja inflación y actividad, lleva a prever unos tipos de interés nulos o negativos, similares a los actuales o incluso más bajos, durante un prolongado periodo de tiempo (Bernal y Santacruz, 2015). Ante esta situación de tipos bajos, parece difícil que las cuentas de resultados puedan mejorar significativamente en cuanto a los márgenes por desintermediación de intereses. 
Aunque la situación de tipos de interés bajos afecta de forma clara a la forma de hacer banca (dañan los rendimientos de los préstamos concedidos bajo esta situación y, por consiguiente, son los préstamos que irán alimentando la entrada de ingresos durante los próximos ejercicios), no podemos olvidar que el negocio bancario se basa en márgenes y, por tanto, si la banda alta del margen se estrecha, la banda baja debe ancharse. Ahí es donde se sitúa una de las estrategias que está siguiendo la banca en cuanto a generación de rentabilidad por la parte baja de la cuenta de resultados y cómo esta situación transitoria evolucionará en el futuro hacia un nuevo modelo de negocio.

\section{GRÁFICO 4 \\ CURVAS DE TIPOS DE INTERÉS DE ESPAÑA Y ALEMANIA \\ (CIERRE DE 2015)}

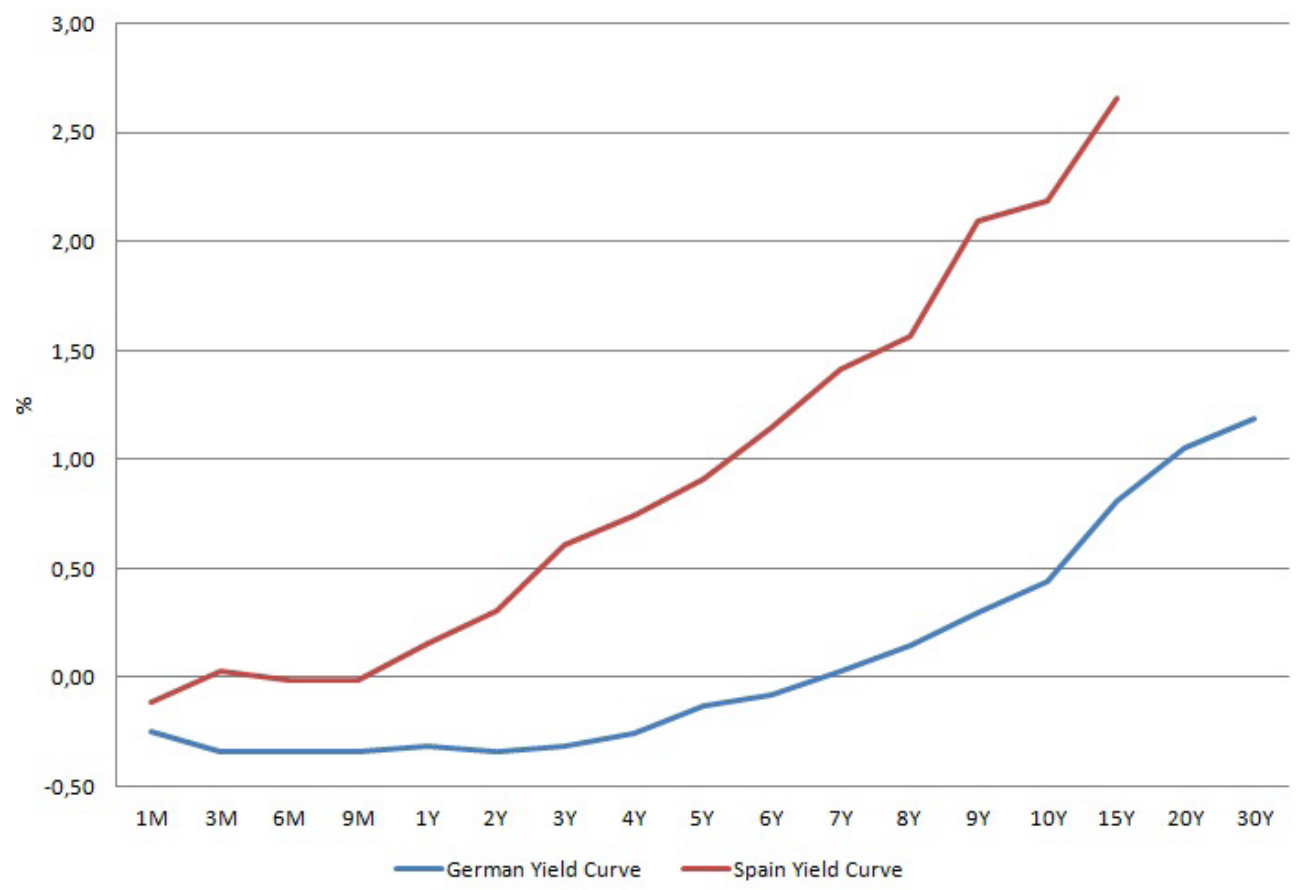

FUENTE: Elaboración propia. Bloomberg.

Por último, dado el impacto en las cuentas de resultados de los bancos, un factor extraordinariamente importante es cuánto puede durar esta situación de tipos de interés bajos. Dado el comportamiento de las curvas de tipos de interés de los soberanos (graficadas anteriormente las de España y Alemania) donde la mayor parte de los bonos de los Tesoros europeos tienen una parte sustancial de su curva de tipos en negativo, sugiere que esta coyuntura se mantendrá a medio plazo. 
Es el caso de Alemania, donde la curva de tipos de interés está en negativo hasta el décimo año por vencimiento, mientras que España tiene también tipos de interés negativos hasta el segundo año, tal como se puede ver en el gráfico anterior. Los países «core» o de la Europa Central -Alemania, Holanda, Bélgica, Austria o Francia- tienen en este momento su curva de tipos de interés en negativo hasta el vencimiento del quinto año mientras que los países rescatados o que están bajo algún tipo de tutela por parte de las autoridades comunitarias (como Grecia o Portugal) tienen toda su curva en positivo. Excepciones a ello son España e Irlanda, con curva en negativo hasta el medio plazo (Irlanda la tiene hasta el segundo año).

\section{Los bancos europeos y el nuevo marco regulatorio: la Unión Bancaria}

Después de la creación de la Unión Monetaria y los avances tanto en unificación monetaria como fiscal, se busca un nuevo diseño institucional más robusto para el sistema financiero y focalizado tanto en la regulación como en la supervisión. Este teórico paso natural desde una unión monetaria a una unificación regulatoria (Santacruz, 2015), la unión bancaria consta de cuatro pilares que adquieren pleno sentido de forma conjunta.

1. Regulación única (single-rule book): Este fue el embrión de la unión bancaria en 2012 con unas reglas de control únicas en materia de supervisión, resolución y garantía de depósitos. En este sentido, las nuevas reglas financieras deberían cumplir con los requisitos de capital exigidos por Basilea III y la regulación de otros aspectos como el consumo de capital en los préstamos y los límites a los salarios de los directivos.

2. Mecanismo Único de Supervisión (SSM por sus siglas en inglés): A través de este mecanismo, el BCE es el supervisor único de 130 bancos cuyo volumen de activos supera los 30.000 millones de euros. Esto deja fuera a un volumen considerable de entidades financieras pequeñas que también deben rendir cuentas ante el BCE.

3. Mecanismo Único de Resolución (SRM por sus siglas en inglés): Este proceso engloba dos vías, las cuales se unen en última instancia. Por un lado, se establece un protocolo de recapitalización de los bancos que lo necesiten mediante un orden de prelación interno asociado a la absorción de pérdidas. Por otro lado, se crea un fondo de rescate con carácter permanente financiado con aportaciones de los bancos y que convivirá en el futuro con el Mecanismo Europeo de Estabilidad (MEDE) para aportar fondos a los bancos con problemas.

El orden de prelación en la absorción de pérdidas no es nuevo: en primer lugar están los accionistas seguidos de los instrumentos de capital híbrido (preferentes, deuda subordinada y CoCos), deuda junior, deuda senior, depósitos no garantizados por el seguro de depósitos y en último lugar el propio fondo de garantía de depósitos. 
4. Fondo de garantía de depósitos común: Por último se busca una unificación de los seguros de depósitos existentes en los países miembros. En este punto todavía no existe un calendario fijo, aunque todo parece indicar que se alargará en el tiempo.

Los cuatro pilares básicos en los que se apoya la unión bancaria, citados anteriormente, tendrían un fin: servir de «cortafuegos» ante futuras crisis bancarias y de solvencia de los Estados poniendo mecanismos que amortigüen el riesgo.

Sobre el papel, la unión bancaria tendría este cometido. Sin embargo, la implementación de este nuevo marco institucional está siendo un proceso lento, costoso y fuertemente distorsionante de la política económica aplicada por cada uno de los socios europeos. Las consecuencias van más allá de las puramente financieras, ya que tanto los ciudadanos como los gobiernos sufrirán cambios determinantes (Santacruz, 2014).

En este punto, los Estados nacionales pierden uno de los objetos más preciados: la regulación de las entidades financieras. A pesar de los acuerdos entre Bancos Centrales y las normas emanadas de dichos acuerdos (especialmente los de Basilea I, II y el III) los gobiernos y los Bancos Centrales nacionales cuentan con un amplio margen discrecional sobre las entidades nacionales de crédito.

Por ejemplo, las normas sobre provisiones, retribución de los accionistas, dotación de reservas de capital, exenciones fiscales por inversiones de gran cuantía o la concesión de fichas bancarias son algunas de las competencias que en este momento están focalizadas a nivel nacional y que, a partir de que ahora, serán controladas por el BCE.

Los primeros pasos dados se encaminan a lograr entidades financieras grandes, con coeficientes de capital sólidos, dotación de provisiones «anticíclicas" y bajo estricto control de las autoridades reguladoras. En este sentido, una de las primeras medidas aprobadas ha sido la limitación del bonus a los directivos bancarios al 100\% del salario fijo. Esta medida intervencionista sobre la política de retribución de los bancos privados ha airado a Reino Unido con cierta legitimidad: los sueldos variables generan incentivos favorables a la consecución de un esfuerzo alto, tal como refleja el problema del principal y el agente.

\section{Basilea III y MiFID II: dos cambios de fondo en la operativa bancaria}

Desde el comienzo de la crisis económica, la actuación de los reguladores bancarios se ha multiplicado en busca de un sistema financiero «más sólido y solvente". Para ello, el «banco de los Bancos Centrales», el Banco Internacional de Pagos de Basilea, endureció los requisitos de capital a la banca según diferentes definiciones, partiendo todas ellas del volumen de activos de riesgo que atesoraran en sus balances (las normas que se conocen con el nombre de Basilea III).

Además de las ratios de capital, Basilea y otras instituciones regulatorias como el Banco Central Europeo (BCE) exigen otro tipo de requerimientos, entre los que se encuentra cuánto capital se consume en las diferentes actividades de crédito. 

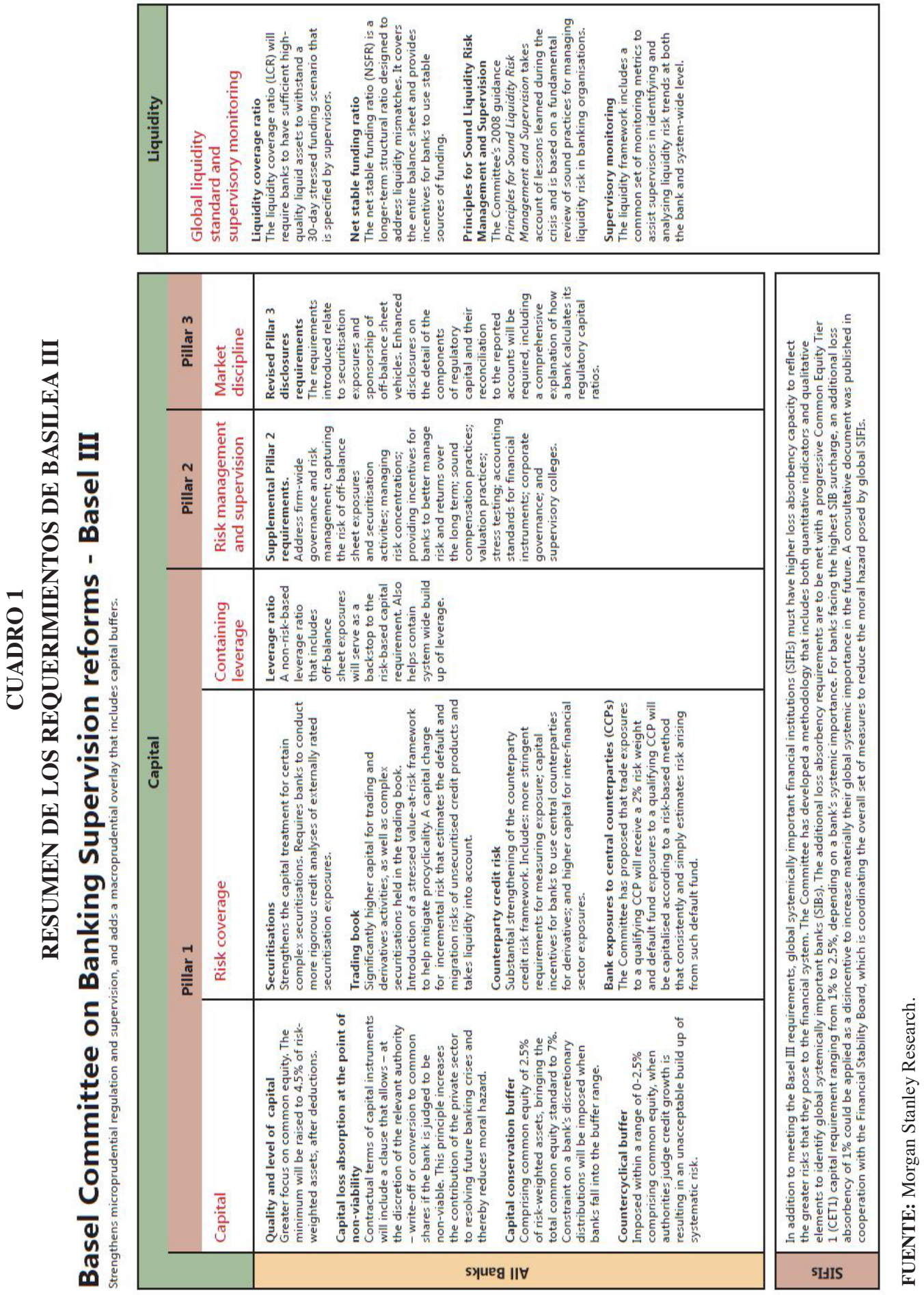
El calendario de implantación de Basilea III se muestra a continuación con los siguientes objetivos.

\section{GRÁFICO 5}

\section{REGULACIÓN SOBRE EL CAPITAL DE ACUERDO CON BASILEA III}

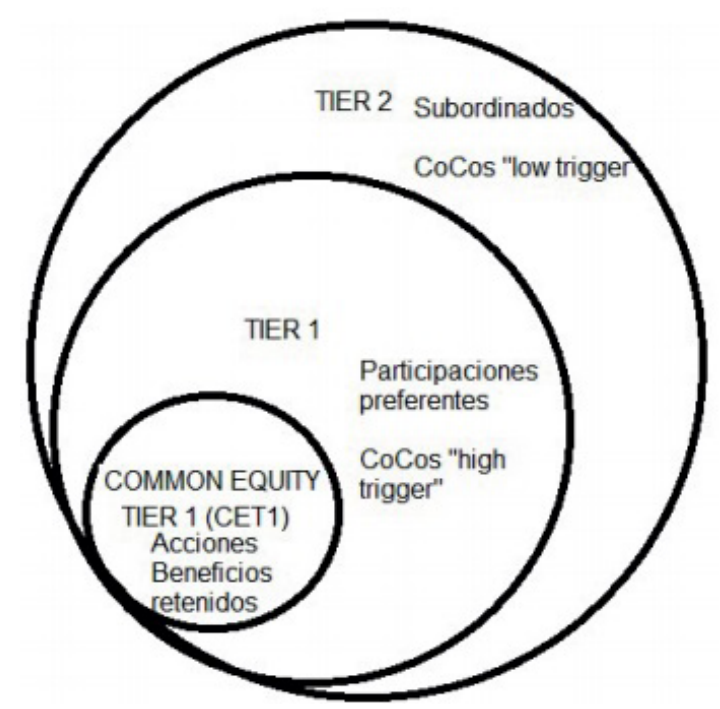

FUENTE: Elaboración propia. BIS.

El calendario está formulado por el Cuadro 2:

\section{CUADRO 2}

\section{CALENDARIO DE IMPLANTACIÓN DE BASILEA III}

\begin{tabular}{|l|l|}
\hline 1 de enero de 2015 & $\begin{array}{l}\text { Consecución de un capital mínimo CET1 del 4,5 por 100, con flexibilidad en } \\
\text { la incorporación de «eligibles» hasta el 1 de enero de 2017 }\end{array}$ \\
\hline $\mathbf{1}$ de enero de 2015 & $\begin{array}{l}\text { Establecimiento de la llamada «ratio de liquidez» con un desfase en salidas } \\
\text { netas de liquidez no superior al 25 por 100 de las salidas brutas }\end{array}$ \\
\hline $\mathbf{1}$ de enero de 2016 & $\begin{array}{l}\text { Creación de un «colchón de conservación del capital» con un mínimo del } \\
\text { 2,5 por 100 más uno opcional denominado «buffer anticíclico» que oscilará } \\
\text { entre el 0 por 100 y el 2,5 por 100 }\end{array}$ \\
\hline $\mathbf{1}$ de enero de 2018 & $\begin{array}{l}\text { Fijación de una ratio de «financiación estable» en sustitución de las ratios } \\
\text { de capital }\end{array}$ \\
\hline
\end{tabular}

NOTA: Todos los porcentajes se miden en términos de activos ponderados por riesgo (RWA).

FUENTE: Elaboración propia a partir de las directrices del BIS (Banco Internacional de Pagos de Basilea). 
En el caso de MiFID II, la normativa sobre Mercados e Instrumentos Financieros, está pendiente de ser traspuesta a las diferentes normativas de los países miembros. En general, se trata de un proceso en el cual el cliente minorista tendrá una mayor protección frente a los agentes proveedores de productos financieros, además de generar obligaciones constantes de reporting y elaboración de perfiles de riesgo (test de conveniencia e idoneidad de los productos financieros según el tipo de cliente).

\section{Modelo de negocio y reestructuración de la banca europea}

En medio de las múltiples incertidumbres ante las que se enfrentan los bancos debido a la situación de la macroeconomía y los cambios regulatorios impulsados y descritos anteriormente, el objetivo último de las partes implicadas es el de crear una industria bancaria blindada frente al riesgo, entendiendo este como el conjunto de sucesos que han llevado a problemas a las entidades en el pasado. Dicho de otra forma, el sistema crediticio nuevo se desarrollaría en un ámbito de aprendizaje de los errores y excesos cometidos en las últimas dos décadas.

Tomando este principio básico que invocan las diferentes innovaciones regulatorias y controles vistos anteriormente (Basilea III, BCE, MiFID II, EBA, stress test, etcétera) conllevan inexorablemente el siguiente resultado: menos riesgo implica necesariamente menos rentabilidad (Knight, 2006 [1921]). En este punto, surgen dos confusiones que aún hoy no se han aclarado: confundir volatilidad con riesgo y riesgo con solvencia. Sin duda, es necesario que los bancos sean más solventes y estén más capitalizados. Eso, en primera ronda, implica menos rentabilidad ya que el denominador del ROE o rentabilidad sobre recursos propios crece (más fondos propios) más rápido de lo que lo hace el numerador (resultado después de impuestos). Esta correlación inversa que se observa en un primer momento (reflejada en el Cuadro 3) no es más que un efecto de corto plazo hasta que el nuevo capital genera «fondo de comercio» suficiente para generar resultados (un banco más sólido es más atractivo para un cliente que un banco que se sepa que tiene problemas de capital).

Sin embargo, a largo plazo no existe tal trade-off entre ser más rentable o ser más solvente. Un banco más capitalizado tiene capacidad de gestionar su negocio de forma más eficiente y prestar servicios de mejor calidad, ya que de esto dependerá su goodwill o su prestigio-marca como palancas de crecimiento en el tiempo. En cambio, sí hay que elegir entre querer más rentabilidad o menos riesgo. 
CUADRO 3

PRINCIPALES INDICADORES A CIERRE DEL PRIMER TRIMESTRE DE 2016 DE LA BANCA EUROPEA

(Datos a 3 de marzo de 2016)

\begin{tabular}{|l|c|c|c|c|c|}
\hline \multicolumn{1}{|c|}{ Entidad } & P/E ratio & Price/Book & ROE $(\boldsymbol{\%})$ & $\begin{array}{c}\text { 1yr Return } \\
(\boldsymbol{\%})\end{array}$ & $\begin{array}{c}\text { CET 1 fully- } \\
\text { loaded }(\boldsymbol{\%})\end{array}$ \\
\hline Banco Santander & 8,13 & 0,59 & 7,78 & $-37,55$ & 12,38 \\
\hline BBVA & 14,55 & 0,77 & 5,17 & $-24,95$ & 12,32 \\
\hline Caixabank & 18,25 & 0,63 & 4,12 & $-31,99$ & 11,77 \\
\hline Bankia & 11,63 & 0,79 & 6,76 & $-23,88$ & 12,92 \\
\hline Banco Popular & 16,63 & 0,39 & 0,83 & $-39,29$ & 12,34 \\
\hline Bankinter & 15,07 & 1,48 & 10,0 & $+0,5$ & 11,82 \\
\hline Banco Sabadell & 10,05 & 0,68 & 5,93 & $-21,13$ & 11,12 \\
\hline Mediobanca & 9 & 0,7 & 8,23 & $-9,93$ & 12,45 \\
\hline BNP Paribás & 7,18 & 0,54 & 7,76 & $-13,39$ & 10,7 \\
\hline UBS & 9,54 & 1,08 & 11,65 & $+0,06$ & 13,7 \\
\hline Credit Suisse & 8,38 & 0,63 & 7,84 & $-11,89$ & 10 \\
\hline Barclays & - & 0,47 & 0,57 & $-26,33$ & 10,6 \\
\hline Lloyd's & 26,8 & 0,91 & 2,71 & $-15,92$ & 12,8 \\
\hline HSBC & 9,37 & 0,68 & 7,66 & $-23,64$ & 11,9 \\
\hline Crédit Agricole & 7,21 & 0,43 & 6,11 & $-18,75$ & 13,9 \\
\hline Deutsche Bank & - & 0,32 & $-6,45$ & $-40,51$ & 11,1 \\
\hline Monte dei Paschi & - & 0,17 & $-40,7$ & $-65,06$ & 11,4 \\
\hline ING & 9,91 & 0,84 & 8,3 & $-11,28$ & 12,7 \\
\hline
\end{tabular}

FUENTE: Elaboración propia. Bloomberg.

En el mundo actual, conforme los tipos de interés de vencimientos cada vez más largos son negativos y las plusvalías de las carteras de renta fija de los bancos se van disipando, las ganancias libres de riesgo ya no existen y, por tanto, cada euro que entre en la cuenta de resultados tendrá asociado un coeficiente de riesgo donde su magnitud no es lo que más importa sino si este riesgo está bien o mal gestionado.

El binomio rentabilidad-riesgo da paso a otra relación no menos importante: retorno frente a coste de capital. En virtud de los números más recientes (Bain\&Co, 2014), el retorno sobre activos ponderados por riesgo (RoRWA, en sus siglas en inglés) mínimo para no destruir valor (es decir, aquel que cubre el coste de capital) ha subido sustancialmente hasta situarse para la banca europea entre el 1,6 por $100 \mathrm{y}$ el 1,8 por 100 de los activos ponderados por riesgo. 


\section{GRÁFICO 6 \\ EVOLUCIÓN DEL RORWA VS. UMBRALES DE COSTE DE CAPITAL (Banca europea)}

Banks in core Europe have not covered their cost of capital over the past five years...

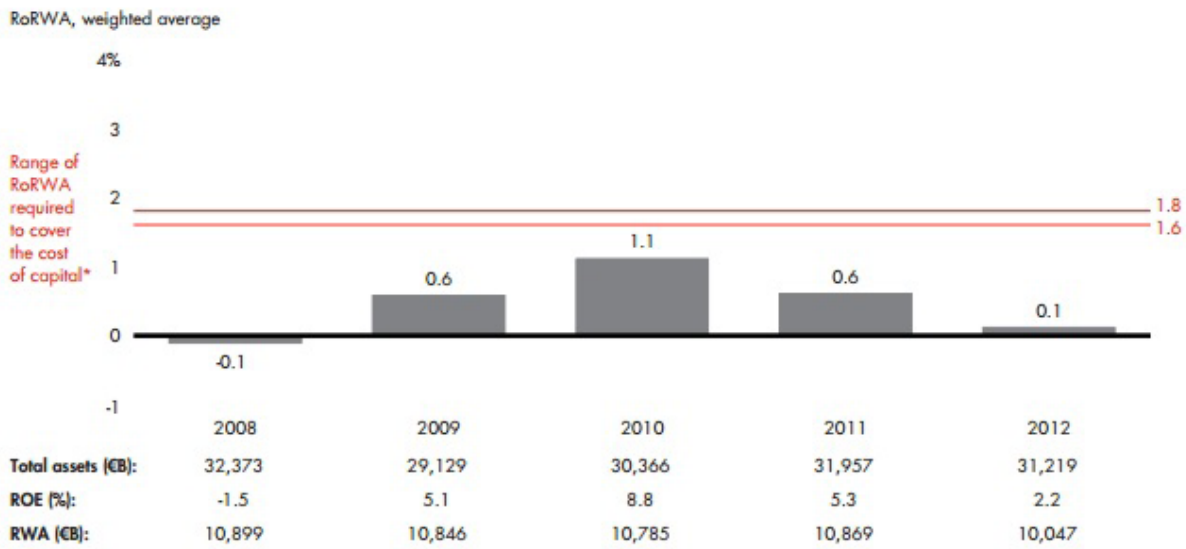

NOTE: * The range represents the level of RaRWA required to cover the banks' cost of capital and varies by bank size and country; analysis of 88 banks based in Austria, Belgium, France, Germany, Ireland, Italy, the Netherlands, the Nordics, Portugal, Spain, Switzerland and the UK.

SOURCE: Banks' financial statement 2008-2012; Bain analysis.

En este sentido, a pesar de que los tipos de interés nulos o negativos reducen teóricamente el coste de capital por la vía de los recursos ajenos, la presión regulatoria, los incentivos que están dando los Bancos Centrales poniendo gratis algo que antes costaba dinero (el propio dinero de curso legal) y la presión de márgenes por un negocio «libre de riesgo», están provocando incrementos del coste de capital que caben traducirse como una elevación del tipo de interés real (precio del consumo presente respecto del futuro) de la banca europea.

Un tipo de interés real mayor no deja de ser una paradoja, ya que conforme los tipos de interés nominales (dictados por los Bancos Centrales en última instancia) siguen cayendo incluyéndose a negativo, los tipos de interés reales de los bancos suben además en ausencia de inflación. Más que una violación de la canónica ecuación de Fischer (aquella que relaciona magnitudes nominales y reales), se trata de una constatación de que los tipos de interés nominales más que responder al verdadero precio del dinero, responden a los deseos de los Bancos Centrales de forzar las expectativas de renta y riqueza a corto plazo para estimular la demanda.

Dicho de otra forma: el tipo de interés real como precio de intercambio de los bienes de una economía entre el presente y el futuro, se distancia cada vez más del tipo de interés nominal, reflejando el riesgo real de la operativa bancaria. Con los actuales ROE y RoRWA, la mayor parte de la banca europea está destruyendo valor en términos promedio, tal como muestran los Gráficos 7 y 8 . 


\section{GRÁFICO 7}

\section{EVOLUCIÓN DEL RORWA VS. UMBRAL DE COSTE DE CAPITAL (10 mayores bancos de Europa hasta 2012)}

\section{Europe's 10 biggest banks come closest to earning their cost of capital}

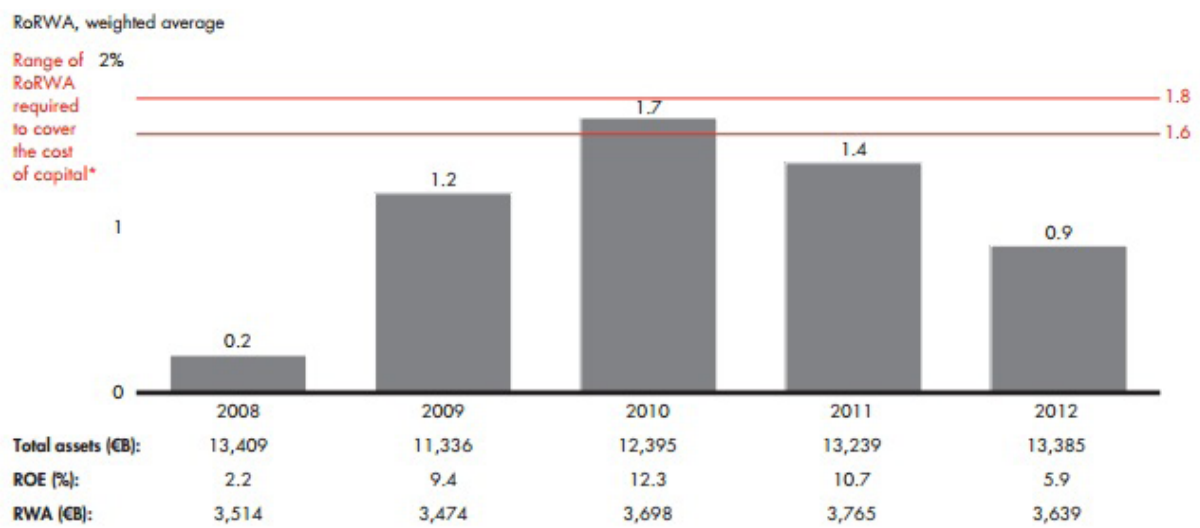

NOTE: * The range represents the lavel of RaRWA required to cover the banks' cost of capital and varies by bank size and country. Analysis of the 10 largest Europe-headquartered banks by market capitalization, with a pan-European presence, a universal bank business model and assets of at least $€ 650$ billion (Barclays, BNP Paribas, Credit Agricole, Credit Suisse, Deutsche Bank, HSBC, Nardea, Santander, UBS and UniCredit.

SOURCE: Banks' financial statement 2008-2012; Bain analysis.

\section{GRÁFICO 8}

\section{EVOLUCIÓN DEL RORWA VS. UMBRAL DE COSTE DE CAPITAL (Otros grandes bancos de Europa hasta 2012)}

Other large European banks have fallen well short of earning their cost of capital

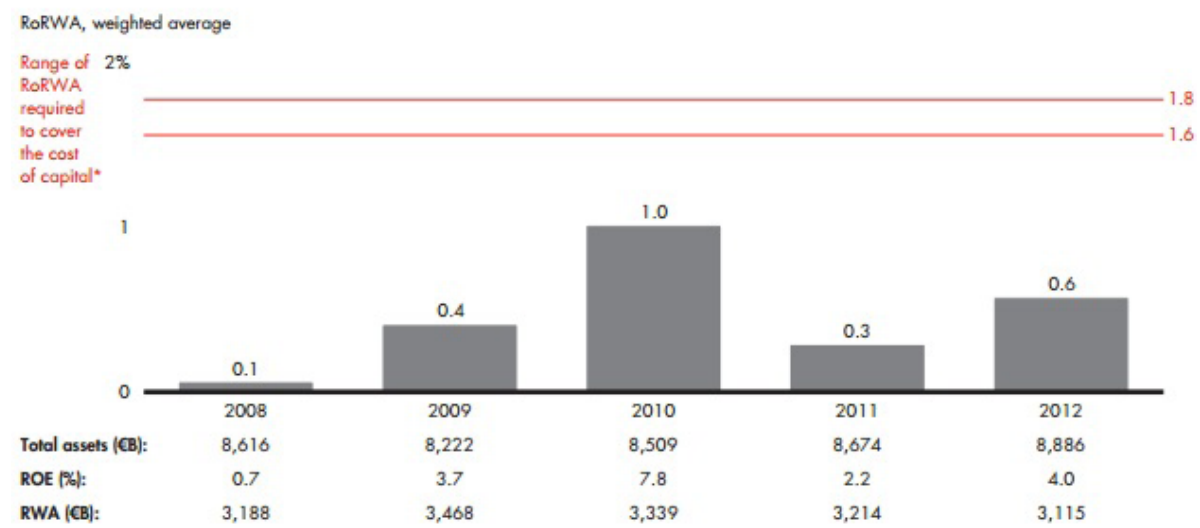

NOTE: * The range represents the lavel of RaRWA required to cover the banks' cost of capital and varies by bank size and country. Analysis of the 13 banks with assests of at least $€ 200$ billion, a universal bank business model and small to medium-sized subsidiaries outside their code geography (ABN AMRO Group, BBVA, Commerzbank, Danske Bank, Erste, ING, Intesa Sanpaolo, KBC, Lloyds, Rabobank, RBS, Société Générale and Standard Chartered).

SOURCE: Banks' financial statement 2008-2012; Bain analysis. 
La destrucción de valor, lejos de ser la causa, es la consecuencia y el reflejo de la necesidad de una reforma en profundidad del modelo de negocio de la banca europea, donde hay pocas entidades que se «salvan de la quema». Es el caso de Nordea, HSBC o BNP Paribas (dentro del grupo de las diez mayores entidades de Europa) u otros como Standard Chartered, Erste o ING, entre otros.

El ajuste y cambio en el modelo de negocio está empezando a echar a andar, especialmente de forma acelerada por la penetración de la tecnología financiera, como veremos en la siguiente sección. Por lo pronto, el periodo transitorio que ha tenido el sistema crediticio entre 2010 (inicio de la crisis de deuda en la Eurozona) y 2015 (inicio del programa de expansión cuantitativa QE) ha llegado a su fin y es el momento de plantear la estrategia a largo plazo.

Insistiendo una vez más en que un banco no trabaja con tipos de interés sino con diferenciales de tipos o márgenes, la fuente de ganancias de un banco (y su modelo de negocio) está en conseguir márgenes amplios: cobrar al cliente más de lo que le cuesta el dinero que recibe. Por tanto, el problema no es que haya tipos negativos sino márgenes muy estrechos como consecuencia de una falta de modelo de negocio agravada por la presión regulatoria.

En este periodo transitorio en medio de operaciones multimillonarias de rescate y configuración del nuevo marco regulatorio, las fuentes de retorno bancario se han ido cayendo conforme se desciende en la cascada de resultados. Acudir al negocio bancario tradicional (margen de intereses + comisiones) ya no es opción puesto que el BCE al mismo tiempo que inyecta dinero de forma masiva y cobra a los bancos que hacen depósitos en él para forzar la circulación del crédito, penaliza la concesión de préstamos catalogados como «riesgo» obligando a provisionar más y limpiar la cartera crediticia de morosidad latente.

\section{GRÁFICO 9}

\section{EVOLUCIÓN DE LA TASA DE MOROSIDAD EN ESPAÑA}

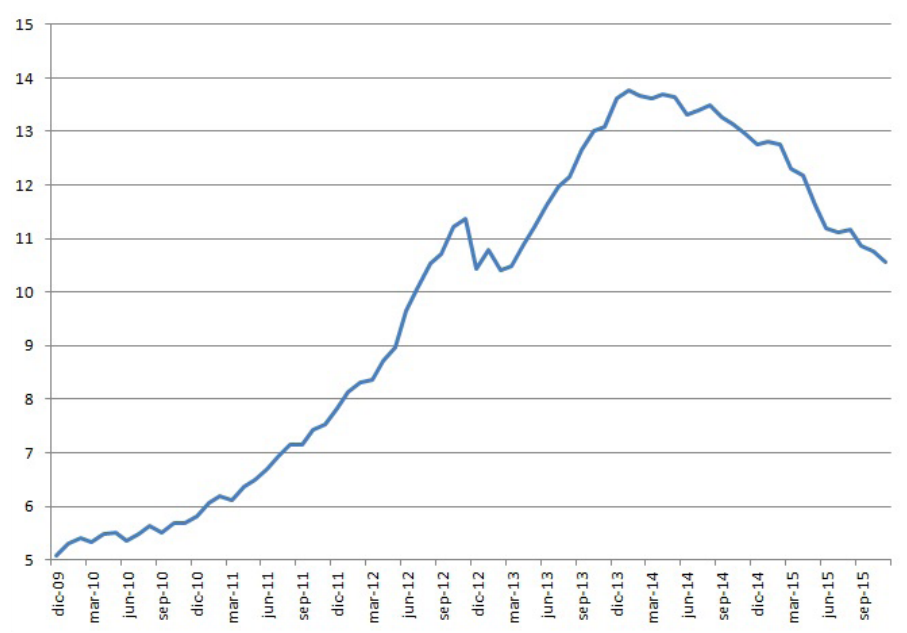

FUENTE: Elaboración propia. Banco de España. 
El proceso de ajuste necesario para purgar los excesos cometidos, ha llevado a la banca española de un extremo a otro en la escala del riesgo: de estar enormemente expuesta a las actividades de mayor riesgo como el crédito promotor a un escenario de riesgo convergente a cero invirtiendo una buena parte de su balance en operaciones de compra y venta de deuda pública y restringiendo todavía el stock de crédito concedido (tal como puede verse en el Gráfico 2) llevándolo a niveles de junio de 2006.

\section{GRÁFICO 10 \\ TASAS DE MOROSIDAD POR BANCOS A CIERRE DE 2015 \\ (Banca española)}

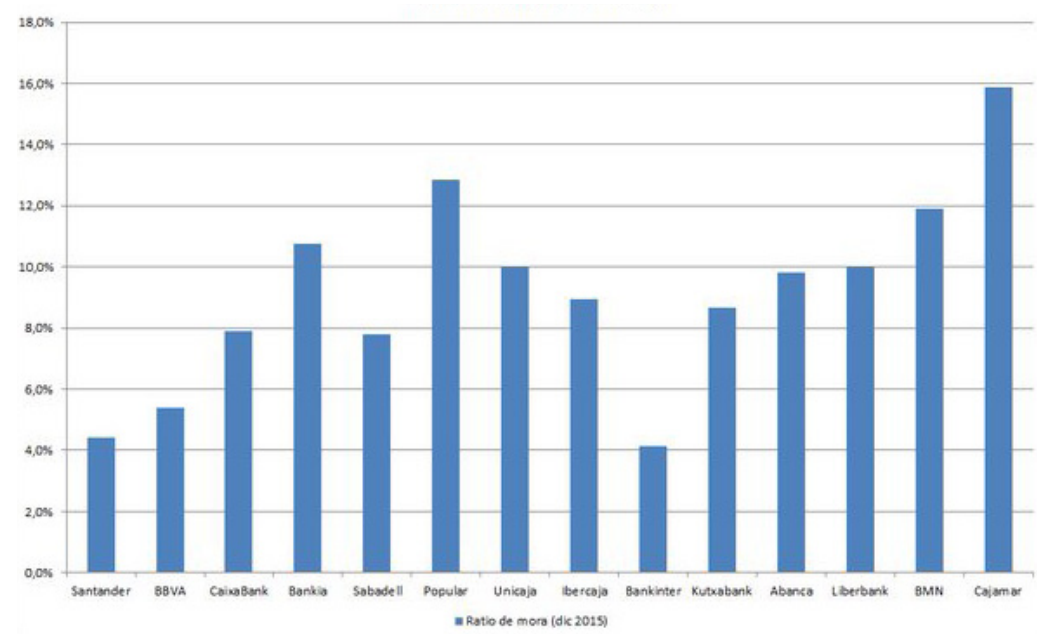

FUENTE: Elaboración propia. Cuentas anuales de los bancos y Banco de España

Conforme vamos descendiendo en la cascada de resultados, tampoco se puede acudir al ROF (resultado por operaciones financieras) o plusvalías por cartera de renta fija ya que se van agotando los títulos que los bancos compraron en el mínimo de precios y máximo de cupones. Llegados a este punto, solamente queda una estrategia usada no solo por el grupo de los 10 bancos más importantes de Europa sino también por el resto: crédito al sector público, operativa en el mercado de renta fija, intermediación de fondos de inversión y fondos de pensiones, recorte de costes y abandono de negocios que consumen más capital en términos relativos como las actividades de banca de inversión, trading en mercados como el de divisas o materias primas y la creación y movimiento de derivados financieros.

Incluso, la operativa con deuda pública ya consume capital según las normas de Basilea III, para evitar la retroalimentación del círculo vicioso entre riesgo soberano y riesgo bancario. Pero mientras Basilea III se implementa de forma total en 2019, 
la banca europea busca «clientes seguros» o que den la apariencia de ello con un margen que todavía es moderado en el peso del ROF sobre el margen típico. Bancos como Santander están incrementando un 12,9 por 100 interanual el crédito al sector público mientras otros como BBVA aumentan casi un 14 por 100 la cartera de renta fija, con datos de cierre de ejercicio 2015.

Precisamente, desde que se puso en marcha el programa TLTRO o préstamos del BCE condicionados a dar crédito, ha supuesto un «efecto sustitución» entre prestar al Estado vía compra de bonos o hacerlo a través de crédito ordinario. A pesar de una mejora notable en cuestiones como el descuento de papel comercial o el crédito hipotecario al sector privado, la banca carga las tintas sobre el crédito público a la espera no de rédito sino como forma de obtener ingresos con riesgo nulo.

En esta dinámica de minimización del riesgo aparente, la batalla a dar es la de la eficiencia. En los últimos años, la ratio de costes sobre ingresos (la forma más habitual de medir la eficiencia) o cuántas unidades de coste se necesitan para generar una unidad de ingreso, ha mejorado sustancialmente tanto por tamaño del banco (adelgazamiento de su estructura) como por su más estricta eficiencia en las operaciones. El Gráfico 11 muestra la eficiencia de la banca española ex-ROF por entidades.

\section{GRÁFICO 11}

RATIO DE EFICIENCIA (EX-ROF)

(Enero-diciembre 2015)

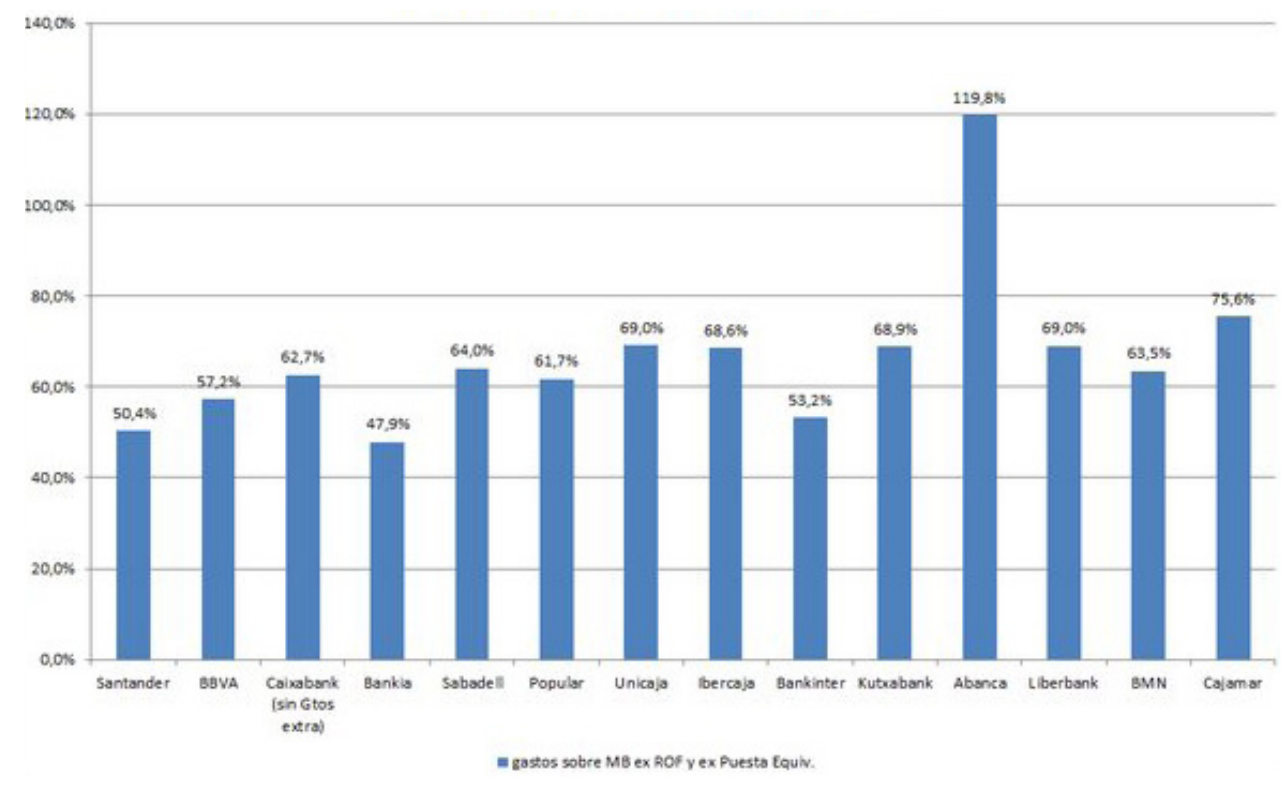

FUENTE: Elaboración propia. Cuentas anuales de los bancos y Banco de España 
¿Es opción subir de una forma importante las comisiones por prestación de servicios? Quizá lo sea, pero hasta el límite tolerable para mantener a la clientela (una clientela que, en muchas ocasiones, ha cambiado depósitos por fondos de inversión). En el fondo, la elección es mucho más profunda, ya que es optar por un modelo en el que el cliente paga por servicio y no por producto y reducir los costes estructurales más rápido de lo que crecen los costes regulatorios en la operativa diaria. Esto puede llevar a una estabilización de los ROE en el entorno del 4-5 por 100 anual promedio con un coste de capital no inferior al 2 por 100.

\section{La industria «fintech»: oportunidades, retos y amenazas para la banca tradicional}

La evolución de la economía y los mercados a nivel mundial, está sirviendo de caldo de cultivo para el desarrollo de nuevas ideas, que intentan satisfacer las necesidades de unos consumidores que se encuentran en constante cambio. A pesar de que la crisis ha sido lenta y dolorosa (ha durado más de siete años), el mercado financiero sigue siendo pionero a la hora de crear nuevos instrumentos y servicios con el objetivo de ganar tanto en eficiencia como en productividad a medio y largo plazo.

Hoy en día el modelo de grandes corporaciones empresariales, no solo financiera sino de cualquier tipo, está cambiando. Las grandes compañías tecnológicas abarcan un gran abanico de campos, lo que les permite ofrecer muchísimos servicios vinculados al conocimiento del cliente y la interrelación que tienen con el mismo.

En este sentido, en los últimos años ha surgido un movimiento cada vez más extendido dentro de la innovación financiera: la aplicación de la tecnología y las telecomunicaciones al mercado financiero (fintech). Hasta el momento, numerosas compañías, startups o emprendedores han apostado por el desarrollo de aplicaciones móviles, programas informáticos y sistemas algorítmicos destinados a proveer a los consumidores y empresas de servicios bancarios tan básicos como transferencias, financiación de circulante o depósitos, además de gestión de carteras y asesoramiento financiero con los denominados robo-advisors.

Fintech es la palabra que se está utilizando para un amplio abanico de empresas que proveen a los clientes de servicios financieros. El origen de la palabra lo dice todo, pues es la unión de finance y technology. Las fintech son empresas que ofrecen una variedad de productos, servicios y soluciones financieras semejantes a los que las entidades bancarias y similares realizan, pero con la características de una mayor agilidad, rapidez e irrupción en un nuevo canal como es el virtual o internet. Es decir, utilizan la tecnología para proporcionar soluciones reales y sencillas a sus clientes a través del canal virtual.

Precisamente, esta canal virtual se erige como un canal de gran crecimiento y máximo desarrollo puesto que las nuevas generaciones, los denominados millenians, 
ya están haciendo uso mayoritario del mismo y se espera que su evolución continúe ganando adeptos. La expresión utilizada por los bancos: los próximos clientes bancarios no pisaran una oficina bancaria, resume a la perfección la revolución que parece estar en marcha.

Pero además del conocimiento y del dominio del canal virtual, en muchos casos las fintech optan por no cobrar por los servicios financieros ofrecidos. En este momento, su estrategia no es conseguir ingresos con los servicios ofertados, sino aumentar el número de usuarios y clientes.

Las plataformas fintech juegan un doble papel en el mercado actual: por un lado, son competidoras del sistema bancario y la gestión tradicional de patrimonios, ya que prestan servicios de alto valor añadido que un banco o una gestora de fondos no pueden realizar. Por otro lado, ejercen como complemento a los productos financieros actuales, proporcionándoles una mayor eficiencia y profundidad de mercado.

Conviene señalar que no existe hoy por hoy una uniformidad en cuanto a una posible clasificación de su actividad. En todo caso señalar que estas empresas constituyen ya una desagregación en toda regla de los servicios bancarios, tal y como puede observarse en el Gráfico 12.

\section{GRAFICO 12}

\section{DESAGREGACIÓN DE LA OFERTA BANCARIA}

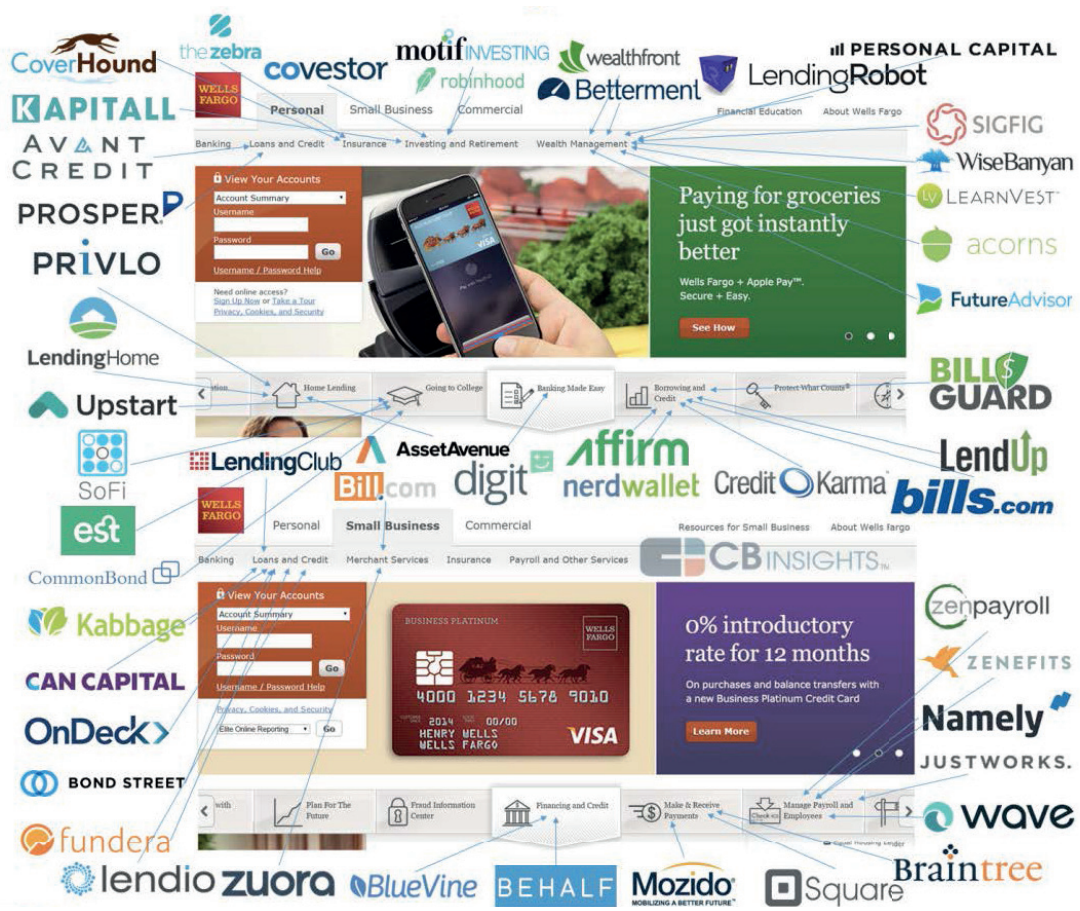

FUENTE: CBInsights. 
Como hemos visto la irrupción de estas nuevas empresas afecta a una variedad de servicios y productos bancarios, tal y como aparece reflejado en el Gráfico 13.

\section{GRAFICO 13 \\ TIPOLOGÍA DE ACTIVIDAD DE LAS EMPRESAS FINTECH}

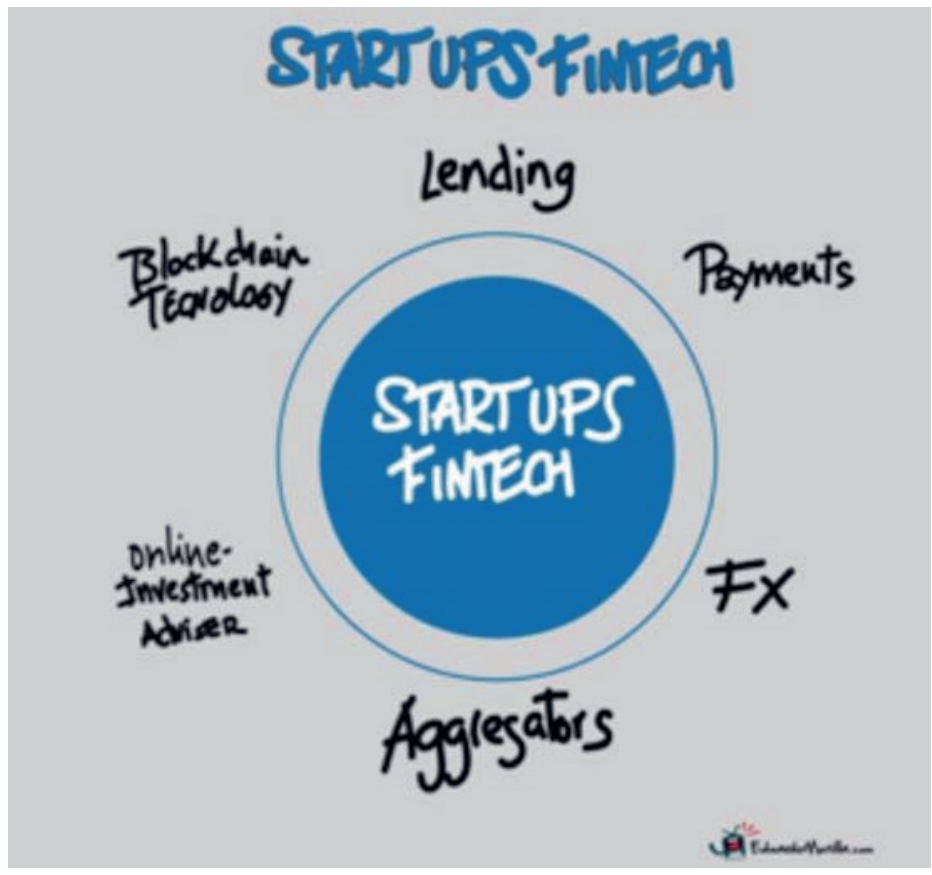

FUENTE: Instituto de Estudios Bursátiles

Básicamente las actividades que realizan en este momento son:

- Lending o crowdlending: dentro de este segmento encaminado a la financiación de empresas y particulares se incluyen toda una serie muy completa de actividades y que son competencia directa actualmente de la banca, más si cabe teniendo presente que hasta hace muy poco los bancos prácticamente eran reacios a prestar. Para hacernos una idea de las prestaciones que incluyen tenemos la compensación de deudas, factoring, $p 2 p$ lending, lending marketplace, direct lending y minicréditos.

- Online Investment Adviser o gestión patrimonial virtual, donde se prestan servicios semejantes a los que ofrecen las diferentes bancas privadas y personales de bancos o gestoras de fondos. En este segmento se integran las plataformas de wealth management, gestores automatizados o roboadvisors, trading algorítmico, social trading, microtrading y crowdfunding inmobiliario. 
- Agregadores y asesoramiento en finanzas personales un servicio que esta relacionado con lo que denominaríamos la gestión de las finanzas personasl o PFM (personal finance management). Aquí la innovación y el surgimiento de nuevas competencias es constantes, algunas de las actividades que podrían señalarse son: comparadores, recomendaciones, buscadores de servicios y productos, herramientas de ahorro y gestión del mismo, programas de seguimiento de la situación financiera de cada usuario.

- Servicios transaccionales, donde agrupa la actividad de transferencias internacionales y cambios de divisa.

- Medios de pago, relacionado con pagos vía móvil o utilizando cualquier otro tipo de canales, dispositivos o validación con la huella digital.

- Tecnología blockchain: actividades relacionadas con Bitcoin y las criptodivisas hasta el momento. Sin embargo, esta es la actividad que para muchos más desarrollos puede atraer en el futuro; de hecho, empresas de la talla de Microsoft están trabajando en grandes desarrollos.

\section{Conclusiones}

Los bancos son conscientes de la fuerte competencia que supone para su negocio, además han interiorizado que deben ir adaptando su negocio a la nueva realidad, así como a los nuevos canales. Es por ello que se puede ver como grandes bancos financian y colaboran que con pequeñas empresas que desarrollan nuevos conceptos o amplían los ya existente dentro del mundo de las fintech. También por supuesto el continuo goteo de alianzas entre bancos y las nuevas empresas fintech.

El objetivo de los bancos es un cambio de estructura desde el modelo actual hacia uno más acorde con el de las grandes empresas tecnológicas que comentábamos anteriormente.

\section{Referencias bibliográficas}

[1] BERNAL ALONSO, M.A y SANTACRUZ CANO, J. (2015). «¿Existe sobrevaloración en la renta fija? Un estudio empírico», Axesor. Departamento de Investigación IEB, WP2015-1011-TA.

[2] CAPACHIN, J. (2010). «Change on the horizon: The impact of cloud computing on treasury and transaction banking». Journal of Payments Strategy \& Systems, 4 (4), 334344.

[3] CASTEJÓN MARTÍN, L. (coord.) (2015). La transformación digital de la banca española. Estudio Banca Digital España 2015, Departamento de Investigación IEB.

[4] D'ACUNTO, R.; OLDRINI, A. y SINN, W. (2013). European Banking: Striking the right balance between risk and return, Bain \& Company Report.

[5] DAPP, T. F.; SLOMKA, L. y HOFFMANN, R. (2015). Fintech reloaded-Traditional banks as digital ecosystems. Deustche Bank Research, March, 2015. 
[6] KAMINSKA, I. (2015). «Fintech: a new power in the land?» Financial World, 4-6.

[7] KNIGHT, F. H. (2006). Uncertainty, Risk and Profit, Dover Publications, New York (republication of 1956 and original in 1921 by the Houghton Mifflin Company, Boston).

[8] LUNN, B. (2014). Google's ambitions in Fintech, Bank Innovation Summit.

[9] MCMANUS, B. (2016). «The Millennial Generation and the Future of Finance: A different kind of trust», Wharton Fintech, 10 ${ }^{\text {th }}$ February, 2016.

[10] PETERS, G. W.; PANAYI, E. y CHAPELLE, A. (2015). Trends in crypto-currencies and blockchain technologies: A monetary theory and regulation perspective. Available at SSRN 2646618.

[11] PwC LUXEMBOURG (2015). FinTech at the forefront of financial innovation.

[12] SANTACRUZ CANO, J. (2014): «El proceso de la Unión Bancaria y sus consecuencias sobre la macro», Expectativas, núm. 17, Civismo Think Tank, pp. 21-28.

[13] SKAN, J.; DICKERSON, J. y MASOOD, S. (2015). «The future of Fintech and banking: Really disrupted or reimagined?», Accenture Strategy, no. 1.

[14] TRELEAVEN, P. (2015). «Financial regulation of FinTech». Journal of Financial Perspectives, 3 (3).

[15] ZHANG, M. (2014). «The age of robo-advisors», The North Western Business Review, $21^{\text {st }}$ November 2014. 